\title{
Numerical Comparisons and Sex-Related Brain Connectivity
}

\author{
Fabiola R. Gómez-Velázquez ${ }^{1}$, Andrés A. González-Garrido ${ }^{1}$, Ricardo A. Salido-Ruiz ${ }^{2}$, Sulema Torres-Ramos ${ }^{2}$, \\ Aurora Espinoza-Valdez ${ }^{2}$, Israel Román-Godínez ${ }^{2}$ \& Erwin R. Villuendas-González ${ }^{3}$ \\ ${ }^{1}$ Instituto de Neurociencias, Universidad de Guadalajara, Jalisco, Mexico \\ ${ }^{2}$ Departamento de Ciencias Computacionales, CUCEI, Universidad de Guadalajara, Jalisco, Mexico \\ ${ }^{3}$ Facultad de Psicología, Universidad Michoacana de San Nicolás Hidalgo, Michoacán, Mexico \\ Correspondence: Fabiola R. Gómez-Velázquez, Instituto de Neurociencias, Universidad de Guadalajara, \\ Francisco de Quevedo 180, Arcos Vallarta, Guadalajara, Jalisco, 44130, Mexico.
}

Received: April 14, 2021

Accepted: May 17, 2021

Online Published: May 20, 2021

doi:10.5539/ijps.v13n2p62

URL: https://doi.org/10.5539/ijps.v13n2p62

\begin{abstract}
Despite the recent literature on sex-related anatomic, maturational and functional brain differences, the study of significant individual developments in math learning and achievement has scarcely approached this perspective. We aimed to compare the influence of sex in functional brain connectivity and behavioral measures in a numerical comparison task. Therefore, a group of school children with ages from 8 to 11 years old was evaluated during a number comparison task. Even though the behavioral performance was similar across the sexes, males distinctly showed a significant correlation between their math WRAT-4 scores and the number of correct responses in the experimental task and working memory scores. Besides, the analysis of the concurrent EEG during task performance showed that males comparatively had a greater brain left intra-hemispheric connectivity, as well as greater interhemispheric connectivity, particularly in Theta and Alpha bands during task performing -as compared to resting-. In contrast, females showed a significantly different decrement of brain connectivity in the Alpha band from resting to task performing. Present results are interpreted as probably reflecting sex-related maturational dissimilarities in neurodevelopment, along with the progressive development of more efficient cognitive strategies, processes running not necessarily parallel in both sexes.
\end{abstract}

Keywords: EEG Coherence, numerical comparison, magnitude representation, math achievement, sex difference, children

\section{Introduction}

Adequate mathematics learning dramatically impacts individuals' lives and careers, increasing competitive functioning and opportunities in a progressively technologized and demanding global economy.

During the last decades, substantial efforts have been made to study and understand the complex math learning process and its neural functioning substrates, including the contribution of several domain-general variables as working memory, reasoning, processing speed, oral language, numerical representation, arithmetical retrieval, among others. As a result, the notion that academic achievement requires coordination between multiple complex cognitive systems has been strengthened (Bryce, Whitebread, \& Szücs, 2015; St Clair-Thompson \& Gathercole, 2006). However, there is an important issue that has remained relatively unattended so far. When math achievement is conceptualized, the sex of the learners is usually not taken into account, despite increasing evidence supporting not only structural differences between sexes but also distinct task-related brain neurofunctional relationships, cognitive abilities, processing strategies, among many others sex-related distinctions.

In general, no apparent sex differences have been consistently demonstrated for the prevalence of the specific calculus-related disorder during neurodevelopment. This disorder, usually known as Developmental Dyscalculia (DD), has been recently defined by Szücs and Goswami (2013) as "persistently weak mathematical performance of developmental origin" when the influence of other factors as motivation or educational appropriateness can be discarded.

Concerning DD, Dirks et al. (2008) reported a slightly higher prevalence for girls than boys in Grade 4 and 5 , while Badian (1999) reported a higher prevalence in boys than girls in Grade 4 and above. The study of Badian 
coincided with results from the Havana Survey (Reigosa-Crespo et al., 2011), in which males showed a higher prevalence of DD than females. In this context, Devine and colleagues (2013) did not find sex differences in the prevalence of DD, but differences emerged when using a mathematics-reading discrepancy definition. Interestingly, these authors found that girls' performance was better in reading than in mathematics, whereas boys' performance was better in mathematics than reading.

Recently, Bull, Cleland, and Mitchell (2013) reported that males have greater acuity of the representation upon spatial illustrations of numbers. They argue that this difference arises from sex-related differences in the parietal lobe. Concerning visuospatial processing, males seem to outperform females primarily in spatial transformation tasks (i.e., mental rotation). Still, they probably depend on different cognitive strategies relying more on imagery and visual processes (e.g., Hugdahl, Thomsen, \& Ersland, 2006), whereas females tend to prefer verbal strategies (Hugdahl et al., 2006). Other studies, using fMRI techniques, have evaluated sex differences in young, healthy subjects while performing verbal and visuospatial working memory tasks. They showed that females exhibit difficulties in both verbal and visuospatial working memory and more significant brain activations interpreted as reflecting lower working memory capacity (Zilles et al., 2016).

An extended correlational study between rates of brain anatomical changes in typically developing adolescents has recently shown that fronto-temporal association cortices have the greater maturational increase in their functional interconnectivity with other cortices (Raznahan et al., 2011). Besides, these authors found evidence for sexually dimorphic maturational coupling within the prefrontal system. They confirmed a close relationship between frontopolar cortex (FPC) and dorsolateral prefrontal cortex (DLPFC) in females but not in males, probably defining sex differences as underlying the neural bases of cognitive control and decision making (Christakou et al., 2009; Raznahan et al., 2011), particularly considering previous reports showing that females tend to have a less lateralized brain organization than males for language (Shaywitz et al., 1995) and spatial abilities (Voyer, Voyer, \& Bryden, 1995).

EEG coherence analysis has demonstrated to be helpful to explore cortical functional relationships, especially when studying the functional coupling between brain areas during cognitive processing (Lacroix et al., 1995; Rappelsberger \& Petsche, 1988; Tremblay et al., 1994). Recently, children showed greater coherence lateralized to the right when they performed a symbolic magnitude comparison task, but with distinctive brain functional connectivity patterns as per the math skill level (González-Garrido et al., 2018).

To the best of our knowledge, this is the first study to assess the brain functional dynamics underlying sex-related differences during a number comparison task's performance. With this aim, we evaluated a group of children to compare the influence of sex in functional brain connectivity while performing a simple numerical task, hypothesizing that sex-related maturational dissimilarities in neurodevelopment will determine significant differences in the brain connectivity patterns underlying the performance of the task despite its behavioral similarities.

\section{Materials and Methods}

\subsection{Participants}

Fifty-seven 8-to-11-year-old, third grade, right-handed children -31 males- participated. All of them completed the math sub-section of the Wide Range Achievement Test (2006). None of the children had been previously reported by their parents or schoolteachers as having difficulties in reading, writing, or calculus.

\subsection{Procedure}

In addition to the WRAT-4, several tests were also applied: the abbreviated IQ version of the Wechsler Intelligence Scale for Children, WISC-IV (Block Design, and Vocabulary), the Edinburgh Inventory of Handedness, the Working Memory Index from the WISC-IV (Digit Span and Letter-Number Sequencing), and a custom-designed survey on neurodevelopmental data to respond by the parents. They were all applied in a single morning session of about 45 minutes, previously obtaining written informed consent from the participants' parents. None of the children had a history of psychiatric or behavioral disorders.

During the experimental session, participants were all comfortably seated in a quiet, dimly lit room, at a $60-\mathrm{cm}$ distance of a 17 -inch LCD monitor (screen resolution= $1280 \mathrm{x}$ 1024) in which visual stimuli were displayed. At the beginning of the session, resting EEG was recorded for three minutes. Children focused on a white fixation-cross displayed in the center of a black background to minimize ocular artifacts. Later, they were instructed to execute a symbolic numerical comparison task.

The experimental task presented pairs of Arabic numerals with values ranging from 5 to 34 . Half of the pairs had a ratio of $1: 2$, while the other half had a higher proportion of $3: 4$. The side on which the number with the higher 
magnitude appeared was counterbalanced. Children had to decide by pressing a key with their left or right index finger, which symbolized a higher quantity. The task was divided into two blocks (56 stimuli each) with a 3 minutes break. The pairs appeared pseudo-randomly within each block, and the order of the blocks was counterbalanced.

Each trial included a fixation cross $(400 \mathrm{~ms})$, followed by the numerical pair $(1000 \mathrm{~ms})$ and a gray screen (400 $\mathrm{ms}$ ). The MINDTRACER software (Neuronic Systems, Inc.) controlled the stimuli' appearance, distinguishing the type of behavioral responses.

\subsection{Stimuli}

One hundred sixty-eight pairs of Arabic numerals were shown on a gray background using yellow and blueprints (54-point Arial font) for the two digits and counterbalancing their spatial -right, left- presentation.

\subsection{Electrophysiological Methods}

\subsubsection{Recording}

The EEG activity was recorded from 19 scalp sites (10/20 international system) using a commercial Electro-Cap and linked earlobes as reference. The Electrooculograms (EOGs) were recorded with surface electrodes from the right eye's outer canthus and infraocular orbital ridge. Inter-electrode impedances were less than five kilo-ohms. A sampling frequency of $200 \mathrm{~Hz}$ and a $0.05-30 \mathrm{~Hz}$ bandpass filter were used for obtaining EEG and EOG signals in a MEDICID-04 system (Neuronic Systems, Inc.). Trials lasting two 1000-ms intervals were further considered for analysis. EEG data were segmented into the initial $1000 \mathrm{~ms}$-prior to the stimulus onset (resting period)-, and the following $1000 \mathrm{~ms}$ starting with the stimulus onset (performing period). EEG epochs with voltage exceeding $100 \mu \mathrm{V}$ on an EEG or EOG channel were automatically rejected. Besides, artifacts were also removed by careful visual inspection.

\subsubsection{Data Analysis}

Signal Processing: Before coherence estimation, EEG data was spatially filtered using a Surface Laplacian solution through a realistic head geometry model (Deng, Winter, Thorpe, \& Srinivasan, 2012). Thus, we built a 3-layer realistic geometry head model of volume conduction. The final spatial sampling was tailored by adjusting the calculations to the averaged coordinates of the EGI 3-D model for children aged 2-9 and 9-18 years, respectively. Subsequently, the connectivity matrix was calculated using the magnitude-squared coherence from surface Laplacian EEG (Nunez et al., 2009; Srinivasan, Winter, Ding, \& Nunez, 2007).

The resulting EEG signals were filtered in delta (0.5-3.5 Hz), theta (3.5-7.5), alpha (8.0-13.5), and beta (14-30 $\mathrm{Hz}$ ) bands, thus reducing the high-frequency noise. Coherence was calculated among the 19 scalp sites only for trials with correct responses; therefore, we obtained 361-coherence spectra symmetric matrix displaying the relationship between all scalp locations. We calculate the mean of the coherence spectra in each frequency band to reduce coherence spectra into coherence coefficients matrices. The ensuing coherence matrices for each frequency band were centered between their minimum and maximum coherence values. Coherence values lower than half of their coherence range were dropped. Afterward, matrices were normalized for their maximum coherence value (not considering values in the diagonal).

To achieve a homogeneous dataset based on the topological analysis of the coherence matrices, we followed two steps; a) the degree of each electrode (i.e., the number of connections that each electrode had with other electrodes) was calculated, and b) children were divided according to their sex and also grouped concerning their WRAT4 scores to balance these two variables across the groups. Then, the Silhouette method was applied to measure the distance among subjects in a group and establish its cohesiveness. The cohesiveness is ranked in the range of $[-1,1]$, where -1 means entirely away from the group, 0 in the limit of belonging to its group, and 1 when entirely belongs to the group. As a result of this analysis, eight individuals were considered outliers and removed from the groups, thus remaining 27 males and 22 females. Next, we removed the youngest and oldest children to obtain 20 males and 20 females. All children had an estimated IQ of 90 or higher, according to the local norms of the WISC-IV Spanish version.

For each one of the selected subjects, their respective coherence matrices in each EEG frequency band (4 by subject) were all averaged to obtain what was called "whole spectrum" (WS). Coherence matrices were averaged to obtain one representative matrix from each contrasting variable [sex, condition (resting and performing), and EEG frequency band]. A threshold ( $\mathrm{th}=0.5$ ) was used to select only the most robust connections. 
Inter and Intra-hemispheric connectivity

The sum of all electrode inter-connections between the right hemisphere and left hemisphere was calculated for each band to quantify inter-hemispheric connections during resting and while performing. The quantification of the intra-hemispheric relationships followed a similar methodology, with only one difference in the last step. Instead of summing all electrode inter-connections between the right and left hemispheres, we calculated the sum of all intra-connections in each hemisphere or each band. For both levels of the experimental condition (resting and performing), the number of connections obtained in each frequency band was normalized, dividing by the maximum number of links in that band.

"Significant" connections

The brain connectivity was analyzed using a Repeated Measures-ANOVA, considering three within-subject factors (Condition (2: resting, performing); Hemisphere (2: left, right); and Frequency band: (5: delta, theta, alpha, beta, and WS); along with 1-between subjects factor (sex). Bonferroni adjustments were performed when needed using a 95-99\% confidence interval. Two thresholds of $\mathrm{p}<0.05$ and $\mathrm{p}<0.01$ were set.

\section{Results}

\subsection{Behavioral Results}

An independent-samples t-test was used to contrast the behavioral results by sex. There were no significant differences between males and females in the number of correct responses, reaction times, or WRAT-4 scores (Table 1).

Table 1. Sociodemographic data and behavioral performing of the experimental task

\begin{tabular}{lllll}
\hline & Males & Females & t(gl) & $\boldsymbol{p}$ \\
\hline Age & $9.0(0.31)$ & $8.9(0.29)$ & $1.055(38)$ & 0.298 \\
Estimated I.Q. & $112.5(12.9)$ & $104.4(13.0)$ & $2.061(38)$ & 0.060 \\
Working memory score & $106.2(10.8)$ & $103.9(15.8)$ & $0.531(38)$ & 0.599 \\
WRAT4 score & $109.5(23.4)$ & $97.9(14.9)$ & $1.879(32)$ & 0.068 \\
Correct Responses (NCT) & $135.1(16.0)$ & $137.8(13.1)$ & $-0.606(38)$ & 0.548 \\
Response Time (NCT) & $724.3(74.5)$ & $733.7(64.5)$ & $-0.424(38)$ & 0.674 \\
\hline
\end{tabular}

Data are expressed in Mean (Standard Deviation). NCT: number comparison task

A Pearson bivariate correlation analysis (2-tailed) was used to further explore the relationship between different behavioral outcomes. We found a statistically significant positive correlation between WRAT-4 scores and the total amount of correct responses while performing the number comparison task in males $(r=0.541, p=0.014)$, but not in females $(r=0.198, p=0.402)$. Similarly, there was a significant correlation between WRAT-4 scores and the working memory scores in males $(r=0.512, \mathrm{p}=0.021)$ but not in females $(\mathrm{r}=0.375, \mathrm{p}=0.104)$.

\subsection{Brain Connectivity}

\subsubsection{Intrahemispheric Connectivity}

The RM-ANOVA showed significant effects only for the Frequency band $\left(\mathrm{F}_{(3,114)}=6.982, p<0.01, \eta^{2}=0.155\right)$, demonstrating that Beta achieved significantly lower connectivity than the other EEG frequency bands $(p<0.01)$. See Figure 1. 


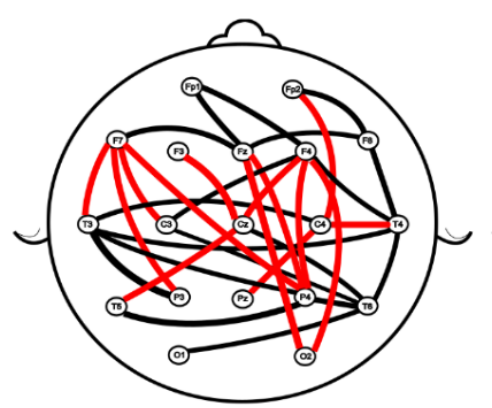

Delta

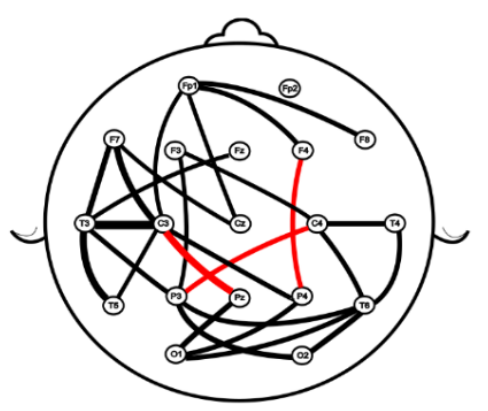

Theta

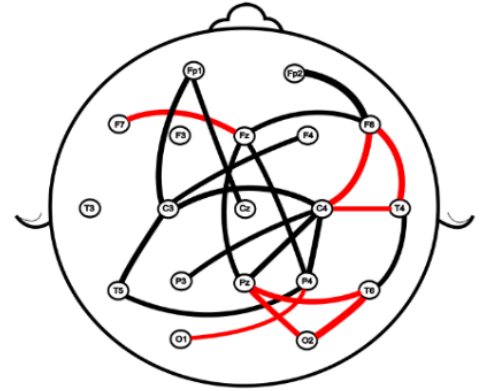

Alpha
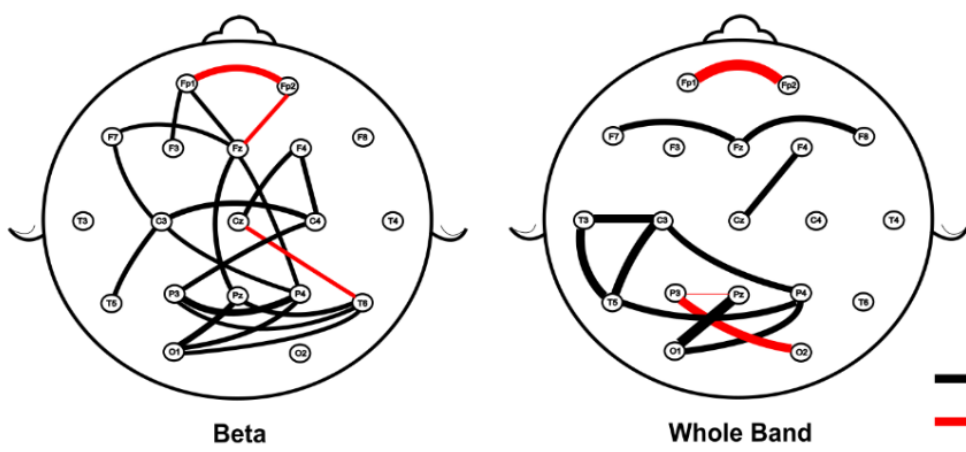

Male > Female

Whole Band

- Female > Male

Figure 1. The graphs show the functional brain predominant organization during task performance, when both sexes are compared. The graph structure was defined by the probability of connections between brain regions, whereas the lines' thickness represents the connectivity's strength

A significant interaction between Condition, Hemisphere and Sex was also found $(F(1,38)=6.612, p=0.041$, $\eta 2=0.148)$. Post hoc analyses showed that differences in brain connectivity between males and females mainly focused on the left hemisphere while individuals performed the task $(p<0.05)$, being males who developed greater brain connectivity during performing as compared to the resting period. Table 2 shows the standardized intrahemispheric connectivity during rest and task performance.

Within-group analyses showed a significant difference between Frequency bands for males $(F(3,57)=3.439, p<$ $0.05, \eta 2=0.153)$, in which Beta reached a significantly lower connectivity index at rest when compared to Alpha $(\mathrm{p}<0.05)$. Brain connectivity difference between the Frequency bands was also observed in females $(\mathrm{F}(3,57)=$ 3.645, $\mathrm{p}<0.05, \eta 2=0.161$ ), who showed significantly lower connectivity in Beta than in Delta $(\mathrm{p}<0.05)$. Furthermore, an additional significant interaction emerged in females between Condition and Frequency band $(\mathrm{F}(3,57)=3.177, \mathrm{p}<0.05, \eta 2=0.143)$, in which post hoc analyses indicated that connectivity in Alpha was significantly lower while performing the numerical task, to that observed during the resting period $(p<0.05)$. Please see Table 2 .

Table 2. Standardized intra-hemispheric connectivity

\begin{tabular}{|c|c|c|c|c|c|c|c|c|c|c|}
\hline & \multicolumn{2}{|c|}{ RESTING } & \multirow[b]{2}{*}{$\boldsymbol{\alpha}$} & \multirow[b]{2}{*}{$\boldsymbol{\beta}$} & \multirow[b]{2}{*}{ WS } & \multicolumn{3}{|c|}{ PERFORMING } & \multirow[b]{2}{*}{$\boldsymbol{\beta}$} & \multirow[b]{2}{*}{ WS } \\
\hline & $\delta$ & $\theta$ & & & & $\delta$ & $\theta$ & $\alpha$ & & \\
\hline \multicolumn{11}{|c|}{ Left Hemisphere } \\
\hline Males & 0.626 & 0.614 & 0.606 & 0.520 & 0.867 & 0.669 & 0.660 & 0.637 & 0.642 & 0.923 \\
\hline Females & 0.625 & 0.604 & 0.620 & 0.482 & 0.885 & 0.644 & 0.549 & 0.483 & 0.533 & 0.882 \\
\hline \multicolumn{11}{|c|}{ Right Hemisphere } \\
\hline Males & 0.671 & 0.593 & 0.657 & 0.526 & 0.886 & 0.648 & 0.603 & 0.638 & 0.544 & 0.871 \\
\hline Females & 0.557 & 0.576 & 0.612 & 0.494 & 0.858 & 0.620 & 0.567 & 0.560 & 0.507 & 0.877 \\
\hline
\end{tabular}

Note: Data reflects a coefficient obtained by dividing the number of significant connections by the number of maximum connections presented for each band. WS: whole spectra. 
Table 3. Standardized inter-hemispheric connectivity

\begin{tabular}{lllllllllll}
\hline & \multicolumn{1}{c}{ RESTING } & \multicolumn{1}{c}{ PERFORMING } \\
& $\boldsymbol{\delta}$ & $\boldsymbol{\theta}$ & $\boldsymbol{\alpha}$ & $\boldsymbol{\beta}$ & WS & $\boldsymbol{\delta}$ & $\boldsymbol{\theta}$ & $\boldsymbol{\alpha}$ & $\boldsymbol{\beta}$ & WS \\
\hline Males & 0.628 & 0.526 & 0.467 & 0.370 & 0.859 & 0.687 & 0.524 & 0.512 & 0.348 & 0.847 \\
Females & 0.551 & 0.461 & 0.364 & 0.322 & 0.800 & 0.598 & 0.422 & 0.395 & 0.290 & 0.780 \\
\hline
\end{tabular}

Note: Data reflects a coefficient obtained by dividing the number of significant connections by the number of maximum connections presented for each band. WS: whole spectra.

\subsubsection{Interhemispheric Connectivity}

Concerning the interhemispheric connectivity, there was a significant effect of the $\operatorname{Sex}(F(1,38)=4.851, p=0.03$, $\eta 2=0.113$ ), in which males showed greater connectivity than females, mainly due to higher connectivity during performing condition, as well as a significant effect for the Frequency band $(F(3,114)=63.183, p<0.001, \eta 2=$ $0.624)$, denoting higher connectivity in Delta $(\mathrm{p}<0.05)$ than Theta, Alpha y Beta. We did not find interactions between the different factors. Table 3 shows the standardized interhemispheric connectivity coefficients during rest and task performance.

\section{Discussion}

Behavioral results showed the absence of sex-related differences between the groups in either the WRAT-4 scores or the accuracy level of responses while performing the experimental task. This result coincides with previous studies obtaining similar cross-sectional findings (Verdine, Golinkoff, Hirsh-Pasek, \& Newcombe, 2017) and longitudinal studies (Vanbinst, Ceulemans, Peters, \& De Smedt, 2018). However, a clear relationship in males was found between WRAT-4 scores and two other behavioral variables: the correct responses while comparing numerical quantities and working memory scores.

A recent neuropsychological evaluation performed in Mexican participants in the birth cohort from an ongoing environmental study called Early Life Exposure in Mexico to Environmental Toxicants (ELEMENT: Perng et al., 2019) studied 826 children from 5 to 15 years old, indicating that males perform significantly better than females on rule-acquisition tasks (i.e., IED: Intra- Extra-Dimensional Shift), which is a test that features visual discrimination and flexibility of attention. Although this result coincides with previously reported sex-related differences in attentional control-processing speed (Anderson, Anderson, Northam, Jacobs, \& Catroppa, 2001), most of the differences are temporary present because girls seem to comparatively move from less accurate to a higher level of performance (especially after 11 years of age), probably suggesting the effect of a sex-specific maturational change in brain development (Giedd et al., 1996; Tomescu et al., 2018). An alternative and not contradicting explanation might assume that each sex employs different strategies while solving a cognitive task (Weissman-Fogel, Moayedi, Taylor, Pope, \& Davis, 2010). That number processing may be related to sex differences in spatial processing strategies (Pletzer, Harris, \& Scheuringer, 2019), while sex hormones relate to hemispheric asymmetries in cognitive functions (Pletzer, Jäger, \& Hawelka, 2019). The association found in males between performances and working memory scores goes in line with recent findings delineating a predictive relationship between executive functions and math abilities, suggesting WM as a "unique predictor of number production and mental calculus achievement" in primary-school children (Filippetti \& Richaud, 2017). Particularly considering that these authors studied a sample of children between 8 and 12 years of age, almost $50 \%$ of the total girls were 8 and 9 years old. In contrast, boys were predominant between 11 and 12 years old.

Roselli and colleagues (2009) have reported that sex seems to predict performances on arithmetical problem-solving tasks. Still, without influence tasks involving number production, a result allegedly since number knowledge is a primary numerical skill, thus less influenced by cultural factors. Empirical evidence links a fluent visual recognition of Arabic digits with math competency development (Price, Wilkeya, \& Yeoab, 2017). However, a combination of visuoperceptual processes, response selection mechanisms, and other cognitive processing steps seem to participate, thus reinforcing the notion that sex-related differences in cognitive processing strategies might play a role in behavioral performance while comparing numerical magnitudes.

Widagdo and colleagues (1998) performed a quantitative EEG analysis to contrast resting with arithmetic processing in young adults. They found a widespread decreased alpha activity with an extensive increase of the activity in delta and theta bands and a focalized posterior increase of the upper beta activity. Recently, Kitaura et al. (2017) used cortical spectral density distribution to compare a mental arithmetic task versus rest and reported a significant increase of the theta activity in medial prefrontal areas with a decrement over the left parietal lobe, 
as well as decreased alphal activity in parietal-occipital regions. These authors demonstrated reduced connectivity within the right hemisphere and significantly increased connectivity in the left hemisphere.

Analogously, present results showed that when performing the number comparison task, males developed greater brain intra-hemispheric connectivity, mainly focused on the left hemisphere. They also showed a higher increase in brain connectivity from resting to performing in both hemispheres. In this sense, it is generally accepted that the comparison of numerical magnitudes is a nonlinguistic activity that relies on both cerebral hemispheres. Indeed, they both can recognize digits and translate them into a mental representation of quantities to be compared (Dehaene, 1996, 1997; Dehaene \& Cohen, 1997). Number comparison might depend on a core system responsible for numerical representations (Feigenson, Dehaene, \& Spelke, 2004). However, the functional brain connectivity and its dynamic change with neural development (Dimitriadis, Laskaris, \& Micheloyannis, 2015), sex seems to be an important additional factor.

A recent EEG study performed in a sex-balanced sample of children from 3rd to 6th grades while solving arithmetic tasks reports a distributed network in the theta band and greater network integration in the alpha band, related to task demands (Vourkas et al., 2014). Regarding our electrophysiological results, sex-related connectivity changes neither run similarly for all the frequency bands; females, for instance, showed a significant decrement for alpha power from resting to task performing in both hemispheres. Several studies have demonstrated the distinct quantitative contribution of the EEG frequency-bands during the performance of mental arithmetical tasks, with a significant role of theta and alpha bands [Kitaura et al., 2017; Chin, Zhang, Wang, \& Ang, 2018; Grabner, \& De Smedt, 2012; Wang, Gan, Zhang, \& Wang, 2018).

On the other hand, current results showed that males developed significantly more inter-hemispheric connections while performing the task, mainly affecting delta and alpha frequency bands. This latter finding might be reflecting the extended bilateral activity over frontal and posterior parietal areas observed during calculation, previously described by using different study methods as EEG (Dimitriadis et al., 2015; Vourkas et al., 2014), MEG (Simos et al., 2008), ERP (Prieto-Corona et al., 2010; Zhou et al., 2011), and fMRI (Kaufmann, Wood, Rubinsten, \& Henik, 2011; Meintjes et al., 2010).

\section{Conclusion}

Whatever the most accurate theoretical explanation might fit the sex-related connectivity differences found, they should be analyzed in the context of the number comparison and mathematical learning in a developing brain (see Peters and De Smedt, 2018 for a detailed and enriching analysis of brain imaging current evidence). Our results could just be reflecting sex-related maturational dissimilarities in neurodevelopment, along with the progressive development of more efficient cognitive strategies, not necessarily concurrent in both sexes.

\section{Contribution of the Present Paper}

Similar behavioral performance with significant sex-related differences in the underlying brain activation patterns while performing cognitive tasks both in healthy (e.g., Bell et al., 2006; Li et al., 2017; Riley et al., 2018) and patients suffering from different pathological conditions (e.g., González-Garrido et al., 2018; Kasher et al., 2019) had been previously reported. These contradictory findings seem to be supported by a sex-related distinct functional brain organization favoring adaptive processing strategies that converge into the goal of maximizing efficient use of task-demanded cognitive resources. It may be the final result of complex genetic-hormone-environment interacting factors leading to preponderant cognitive processing strategies in adults. The present work highlights the role of early sex-related brain functional connectivity differences in mathematical learning and how they distinctly influence numerical operations. Current results reinforce the need to distinguishing sex as a relevant factor to further evaluate math skills in children.

\section{Limitations}

Despite the limitations of the present study, mainly associated with the size of the final sample, resultant sex-related differences in the relationships between several behavioral variables, and the EEG frequency band distribution of the changes from rest to task-performing, along with the sex-related dissimilarities in brain connectivity patterns when comparing Arabic numbers, strongly suggest the need to study this relevant topic further, probably via evaluating populations with a significantly higher range of age, including young adults.

\section{Statements}

\subsection{Acknowledgement}

The authors wish to thank the collaboration of all the children, parents and schools who participated in the study, and Alicia Martínez Ramos for collecting the data. 


\subsection{Statement of Ethics}

The Instituto de Neurociencias Ethics committee on human research approved the study protocol.

\subsection{Disclosure Statement}

The authors have no conflicts of interest to declare.

\subsection{Funding Sources}

This research received no specific grant from any funding agency in the public, commercial, or not-for-profit sectors.

\subsection{Authors Contributions}

FRGV and AAGG served as co-first authors and contributed equally to the paper. FRGV contributed to the conception, design of the feasibility study, acquisition, analysis, interpretation, and manuscript drafting. AAGG contributed to the conception, interpretation, and drafting of the manuscript. RASR, STR, AEV, and IRG contributed to the development, analysis, interpretation and agree to be accountable for all other aspects of the work, ensuring integrity and accuracy. ERVG participated in the re-analysis of the data and final interpretation of the results, also reviewing the manuscript. All authors gave final approval of the manuscript.

\section{References}

Anderson, V. A., Anderson, P., Northam, E., Jacobs, R., \& Catroppa, C. (2001). Development of executive functions through late childhood and adolescence in an Australian sample. Developmental Neuropsychology, 20,385-406. https://doi.org/10.1207/S15326942DN2001_5

Arán Filippetti, V., \& Richaud, M. C. (2017). A structural equation modeling of executive functions, IQ and mathematical skills in primary students: Differential effects on number production, mental calculus and $\begin{array}{lllll}\text { arithmetical } & \text { phoblems. } & \text { Child } & \text { Neuropsychology, } & \text { 23, }\end{array}$ https://doi.org/10.1080/09297049.2016.1199665

Badian, N. (1999). Persistent arithmetic, reading, or arithmetic and reading disability. Annals of Dyslexia, 49, 43-70. https://doi.org/10.1007/s11881-999-0019-8

Bell, E. C., Willson, M. C., Wilman, A. H., Dave, S., \& Silverstone, P. H. (2006). Males and females differ in brain activation during cognitive tasks. Neuroimage, 30(2), 529-538. https://doi.org/10.1016/j.neuroimage.2005.09.049

Bryce, D., Whitebread, D., \& Szücs, D. (2015). The relationships among executive functions, metacognitive skills and educational achievement in 5- and 7-year-old children. Metacognition and Learning, 10, 181-198. https://doi.org/10.1007/s11409-014-9120-4

Bull, R., Cleland, A. A., \& Mitchell, T. (2013). Sex differences in the spatial representation of number. Journal of Experimental Psychology. General, 142(1), 181-192. https://doi.org/10.1037/a0028387

Chin, Z. Y., Zhang, X., Wang, C., \& Ang, K. K. (2018). EEG-based discrimination of different cognitive workload levels from mental arithmetic. Annual International Conference of the IEEE Engineering in Medicine and Biology Society, 2018, 1984-1987. https://doi.org/10.1109/EMBC.2018.8512675

Christakou, A., Halari, R., Smith, A. B., Ifkovits, E., Brammer, M., \& Rubia, K. (2009). Sex-dependent age modulation of frontostriatal and temporo-parietal activation during cognitive control. Neuroimage, 48(1), 223-236. https://doi.org/10.1016/j.neuroimage.2009.06.070

Dehaene, S. (1996). The organization of brain activations in number comparison: event-related potentials and the additive-factors method. Journal of Cognitive Neuroscience, 8, 47-68. https://doi.org/10.1162/jocn.1996.8.1.47

Dehaene, S. (1997). The number sense. Oxford: Oxford University Press.

Dehaene, S., \& Cohen, L. (1997). Cerebral pathways for calculation: double dissociation between rote verbal and quantitative knowledge of arithmetic. Cortex, 33, 219-250. https://doi.org/10.1016/S0010-9452(08)70002-9

Deng, S., Winter, W., Thorpe, S., \& Srinivasan, R. (2012). Improved surface Laplacian estimates of cortical potential using realistic models of head geometry. IEEE Transactions on Bio-medical Engineering, 59(11), 2979-2985. https://doi.org/10.1109/TBME.2012.2183638 
Devine, A., Soltész, F., Nobes, A., Goswami, U., \& Szücs, D. (2013). Gender differences in developmental dyscalculia depend on diagnostic criteria. Learning and Instruction, 27, 31-39. https://doi.org/10.1016/j.learninstruc.2013.02.004

Dimitriadis, S. I., Laskaris, N. A., \& Micheloyannis, S. (2015). Transition dynamics of EEG-based network microstates during mental arithmetic and resting wakefulness reflects task-related modulations and developmental changes. Cognitive Neurodynamics, 9, 371-387. https://doi.org/10.1007/s11571-015-9330-8

Dirks, E., Spyer, G., van Lieshout, E. C., \& de Sonneville, L. (2008). Prevalence of combined reading and arithmetic disabilities. Journal of Learning Disabilities, 41(5), 460-473. https://doi.org/10.1177/0022219408321128

Feigenson, L., Dehaene, S., \& Spelke, E. (2004). Core systems of number. Trends in Cognitive Sciences, 8, 307-314. https://doi.org/10.1016/j.tics.2004.05.002

Giedd, J. N., Snell, J. W., Lange, N., Rajapakse, J. C., Casey, B. J., Kozuch, P. L., Vaituzis, A. C., Vauss, Y. C., Hamburger, S. D., Kaysen, D., \& Rapoport, J. L. (1996). Quantitative magnetic resonance imaging of human brain development: Ages 4-18. Cerebral Cortex, 6(4), 551-560. https://doi.org/10.1093/cercor/6.4.551

González-Garrido, A. A., Gallardo-Moreno, G. B., Romo-Vázquez, R., Vélez-Pérez, H., Flores-Saiffe-Farías, A., Mendizabal-Ruiz, G., Santos-Arce, S. R., Ruiz-Stovel, V. D., Gómez-Velázquez, F. R., \& Ramos-Loyo, J. (2018). Is sex an influential factor in type-1 diabetes neurofunctional development? A preliminary study. Journal of Neuroscience Research, 96(10), 1699-1706. https://doi.org/10.1002/jnr.24268

González-Garrido, A. A., Gómez-Velázquez, F. R., Salido-Ruiz, R. A., Espinoza-Valdez, A., Vélez-Pérez, H., Romo-Vazquez, R., Gallardo-Moreno, G. B., Ruiz-Stovel, V. D., Martínez-Ramos, A., \& Berumen, G. (2018). The analysis of EEG coherence reflects middle childhood differences in mathematical achievement. Brain and Cognition, 124, 57-63. https://doi.org/10.1016/j.bandc.2018.04.006

Grabner, R. H., \& De Smedt, B. (2012). Oscillatory EEG correlates of arithmetic strategies: a training study. Frontiers in Psychology, 3, 428. https://doi.org/10.3389/fpsyg.2012.00428

Hugdahl, K., Thomsen, T., \& Ersland, L. (2006). Sex differences in visuo-spatial processing: an fMRI study of mental rotation. Neuropsychologia, 44(9),

1575-1583. https://doi.org/10.1016/j.neuropsychologia.2006.01.026

Kasher, N., Wittbrodt, M. T., Alam, Z. S., Lima, B. B., Nye, J. A., Campanella, C., Ladd, S., Hammadah, M., Shah, A. J., Raggi, P., Quyyumi, A. A., Vaccarino, V., \& Bremner, J. D. (2019). Sex differences in brain activation patterns with mental stress in patients with coronary artery disease. Biology of Sex Differences, 10(1), 35. https://doi.org/10.1186/s13293-019-0248-4

Kaufmann, L., Wood, G., Rubinsten, O., \& Henik, A. (2011). Meta-analyses of developmental fMRI studies investigating typical and atypical trajectories of number processing and calculation. Developmental Neuropsychology, 36, 763-787. https://doi.org/10.1080/87565641.2010.549884

Kitaura, Y., Nishida, K., Yoshimura, M., Mii, H., Katsura, K., Ueda, S., Ikeda, S., Pascual-Marqui, R. D., Ishii, R., \& Kinoshita, T. (2017). Functional localization and effective connectivity of cortical theta and alpha oscillatory activity during an attention task. Clinical Neurophysiology Practice, 2, 193-200. https://doi.org/10.1016/j.cnp.2017.09.002

Lacroix, D., Chaput, Y., Rodriguez, J. P., Filion, M., Morrison, D., St-Denis, P., \& Albert, J. M. (1995). Quantified EEG changes associated with a positive clinical response to clozapine in schizophrenia. Progress in Neuro-psychopharmacology \& Biological Psychiatry, 19(5), 861-876. https://doi.org/10.1016/0278-5846(95)00116-D

Li, L., Cazzell, M., Zeng, L., \& Liu, H. (2017). Are there gender differences in young vs. aging brains under risk decision-making? An optical brain imaging study. Brain Imaging and Behavior, 11(4), 1085-1098. https://doi.org/10.1007/s11682-016-9580-z

Meintjes, E. M., Jacobson, S. W., Molteno, C. D., Gatenby, J. C., Warton, C., Cannistraci, C. J., Gore, J. C., \& Jacobson, J. L. (2010). An fMRI study of magnitude comparison and exact addition in children. Magnetic Resonance Imaging, 28(3), 351-362. https://doi.org/10.1016/j.mri.2009.11.010 
Nunez, P. L., Silberstein, R. B., Shi, Z., Carpenter, M. R., Srinivasan, R., Tucker, D. M., Doran, S. M., Cadusch, P. J., \& Wijesinghe, R. S. (1999). EEG coherency II: experimental comparisons of multiple measures. Clinical Neurophysiology, 110(3), 469-486. https://doi.org/10.1016/S1388-2457(98)00043-1

Perng, W., Tamayo-Ortiz, M., Tang, L., Sánchez, B. N., Cantoral, A., Meeker, J. D., Dolinoy, D. C., Roberts, E. F., Martinez-Mier, E. A., Lamadrid-Figueroa, H., Song, P. X. K., Ettinger, A. S., Wright, R., Arora, M., Schnaas, L., Watkins, D. J., Goodrich, J. M., Garcia, R. C., Solano-Gonzalez, M... Peterson, K. E. (2019). Early Life Exposures in Mexico to Environmental Toxicants (ELEMENT). BMJ Open, 9, e030427. https://doi.org/10.1136/bmjopen-2019-030427

Peters, L., \& De Smedt, B. (2018). Arithmetic in the developing brain: A review of brain imaging studies. Developmental Cognitive Neuroscience, 30, 265-279. https://doi.org/10.1016/j.den.2017.05.002

Pletzer, B., Harris, T., \& Scheuringer, A. (2019). Sex differences in number magnitude processing strategies are mediated by spatial navigation strategies: evidence from the unit-decade compatibility effect. Frontiers in Psychology, 10, 229. https://doi.org/10.3389/fpsyg.2019.00229

Pletzer, B., Jäger, S., \& Hawelka, S. (2019). Sex hormones and number processing. Progesterone and testosterone relate to hemispheric asymmetries during number comparison. Hormones and Behavior, 115, 104553. https://doi.org/10.1016/j.yhbeh.2019.07.001

Price, G. R., Wilkeya, E. D., \& Yeoab, D. J. (2017). Eye-movement patterns during nonsymbolic and symbolic numerical magnitude comparison and their relation to math calculation skills. Acta Psychologica, 176, 47-57. https://doi.org/10.1016/j.actpsy.2017.03.012

Prieto-Corona, B., Rodríguez-Camacho, M., Silva-Pereyra, J., Marosi, E., Fernández, T., \& Guerrero, V. (2010). Event-related potentials findings differ between children and adults during arithmetic-fact retrieval. Neuroscience Letters, 468, 220-224. https://doi.org/10.1016/j.neulet.2009.10.094

Rappelsberger, P., \& Petsche, H. (1988). Probability mapping: power and coherence analysis of cognitive processes. Brain Topography, 1(1), 46-54. https://doi.org/10.1007/BF01129339

Raznahan, A., Lerch, J. P., Lee, N., Greenstein, D., Wallace, G.L., Stockman, M., Clasen, L., Shaw, P. W., \& Giedd, J. N. (2011). Patterns of coordinated anatomical change in human cortical development: a longitudinal neuroimaging study of maturational coupling. Neuron, 72(5), 873-884. https://doi.org/10.1016/j.neuron.2011.09.028

Reigosa-Crespo, V., Valdés-Sosa, M., Butterworth, B., Estévez, N., Rodríguez, M., Santos, E., Torres, P., Suárez, R., \& Lage, A. (2011). Basic numerical capacities and prevalence of developmental dyscalculia: the Havana Survey. Developmental Psychology, 48(1), 123-135. https://doi.org/10.1037/a0025356

Riley, J. D., Chen, E. E., Winsell, J., Davis, E. P., Glynn, L. M., Baram, T. Z., Sandman, C. A., Small, S. L., \& Solodkin A. (2018). Network specialization during adolescence: Hippocampal effective connectivity in boys and girls. Neuroimage, 2175, 402-412. https://doi.org/10.1016/j.neuroimage.2018.04.013

Rosselli, M., Ardila, A., Matute, E., \& Inozemtseva, O. (2009). Gender differences and cognitive correlates of mathematical skills in school-aged children. Child Neuropsychology, 15, 216-231. https://doi.org/10.1080/09297040802195205

Shaywitz, B. A., Shaywitz, S. E., Pugh, K. R., Constable, R. T., Skudlarski, P., Fulbright, R. K., Bronen, R. A., Fletcher, J. M., Shankweiler, D. D., Katz, L., \& Gore, J. C. (1995). Sex differences in the functional organization of the brain for language. Nature, 373, 607-609. https://doi.org/10.1038/373607a0

Simos, P. G, Kanatsouli, K., Fletcher, J. M., Sarkari, S., Juranek, J., Cirino, P., Passaro, A., \& Papanicolaou, A. C. (2008). Aberrant spatiotemporal activation profiles associated with math difficulties in children: a magnetic source imaging study. Neuropsychology, 22, 571-584. https://doi.org/10.1037/0894-4105.22.5.571

Srinivasan, R., Winter, W. R., Ding, J., \& Nunez, P. L. (2007). EEG and MEG coherence: measures of functional connectivity at distinct spatial scales of neocortical dynamics. Journal of Neuroscience Methods, 166(1), 41-52. https://doi.org/10.1016/j.jneumeth.2007.06.026

St Clair-Thompson, H. L., \& Gathercole, S. E. (2006). Executive functions and achievements in school: Shifting, updating, inhibition, and working memory. Quarterly Journal of Experimental Psychology, 59(4), 745-759. https://doi.org/10.1080/17470210500162854

Szücs, D., \& Goswami, U. (2013). Developmental dyscalculia: Fresh perspectives. Trends in Neuroscience and Education, 2(2), 33-37. https://doi.org/10.1016/j.tine.2013.06.004 
Tomescu, M. I., Rihs, T. A., Rochas, V., Hardmeier, M., Britz, J., Allali, G., Fuhr, P., Eliez, S., \& Michel, C. M. (2018). From swing to cane: Sex differences of EEG resting-state temporal patterns during maturation and aging. Developmental Cognitive Neuroscience, 31, 58-66. https://doi.org/10.1016/j.dcn.2018.04.011

Tremblay, M., Lacroix, D., Chaput, Y., Fraile, V., Lamer, R., \& Albert, J. M. (1994). Brain activation with a maze test: an EEG coherence analysis study in healthy subjects. Neuroreport, 5(18), 2449-2453. https://doi.org/10.1097/00001756-199412000-00011

Vanbinst, K., Ceulemans, E., Peters, L., Ghesquière, P., \& De Smedt, B. (2018). Developmental trajectories of children's symbolic numerical magnitude processing skills and associated cognitive competencies. Journal of Experimental Child Psychology, 166, 232-250. https://doi.org/10.1016/j.jecp.2017.08.008

Verdine, B. N., Golinkoff, R. M., Hirsh-Pasek, K., \& Newcombe, N. S. (2017). V. Results-individual difference factors in spatial and mathematical skills. Monographs of the Society for Research in Child Development, 82(1), 81-88. https://doi.org/10.1111/mono.12284

Vourkas, M., Karakonstantaki, E., Simos, P. G., Tsirka, V., Antonakakis, M., Vamvoukas, M., Stam, C., Dimitriadis, S., \& Micheloyannis, S. (2014). Simple and difficult mathematics in children: A minimum spanning tree EEG network analysis. Neuroscience Letters, 576, 28-33. https://doi.org/10.1016/j.neulet.2014.05.048

Voyer, D., Voyer, S., \& Bryden, M. P. (1995). Magnitude of sex differences in spatial abilities: a meta-analysis and consideration of critical variables. Psychological Bulletin, 117, 250-270. https://doi.org/10.1037/0033-2909.117.2.250

Wang, L., Gan, J. Q., Zhang L., \& Wang, H. (2018). Differential recruitment of brain networks in single-digit addition and multiplication: Evidence from EEG oscillations in theta and lower alpha bands. International Journal of Psychophysiology, 128, 81-92. https://doi.org/10.1016/j.ijpsycho.2018.04.005

Weissman-Fogel, I., Moayedi, M., Taylor, K. S., Pope, G., \& Davis, K. D. (2010). Cognitive and default-mode resting state networks: Do male and female brains "rest" differently? Human Brain Mapping, 31(11), 1713-1726. https://doi.org/10.1002/hbm.20968

Widagdo, M. M., Pierson, J. M., \& Helme, R. D. (1998). Age-related changes in qEEG during cognitive tasks. The International Journal of Neuroscience, 95, 63-75. https://doi.org/10.3109/00207459809000650

Wilkinson, G. S., \& Robertson, G. J. (2006). Wide Range Achievement Test (WRAT4). Lutz, FL: Psychological Assessment Resources. https://doi.org/10.1037/t27160-000

Zhou, X., Booth, J. R., Lu, J., Zhao, H., Butterworth, B., Chen, C., \& Dong, Q. (2011). Age-independent and age-dependent neural substrate for single-digit multiplication and addition arithmetic problems. Developmental Neuropsychology, 36, 338-352. https://doi.org/10.1080/87565641.2010.549873

Zilles, D., Lewandowski, M., Vieker, H., Henseler, I., Diekhof, E., Melcher, T., Keil, M., \& Gruber, O. (2016). Gender differences in verbal and visuospatial working memory performance and networks. Neuropsychobiology, 73(1), 52-63. https://doi.org/10.1159/000443174

\section{Copyrights}

Copyright for this article is retained by the author(s), with first publication rights granted to the journal.

This is an open-access article distributed under the terms and conditions of the Creative Commons Attribution license (http://creativecommons.org/licenses/by/4.0/). 